-研究报告・

\title{
中国东部海岛维管植物的beta多样性及其驱动因素
}

\author{
刘翔宇 ${ }^{1,2}$ 赵慈良 $^{2}$ 许 山 $^{1,2}$ 梁启明 1,2 朱晓粀 1,2 李 亮 ${ }^{1,2}$ 阎恩荣 $1,2^{*}$ \\ 1 (华东师范大学生态与环境科学学院, 上海 200241) \\ 2 (浙江普陀山森林生态系统定位观测研究站, 浙江舟山 316100)
}

\begin{abstract}
摘要: beta多样性描述群落物种组成如何随环境梯度而变化。海岛具有边界清晰、面积和离岸距离不同以及环境变 化剧烈等自然禀赋。目前, 我们对离岸距离、岛间距离和气候因素在海岛植物beta多样性变化格局中的相对贡献 仍认识不足。本研究基于中国东部 36 个海岛的维管植物物种名录, 以Jaccard相异性指数度量beta多样性, 利用 Mantel偏相关分析和beta多样性的变异分解, 探究了海岛不同生活型维管植物的beta多样性格局及其非生物影响 因素。结果显示: 36 个海岛共记录维管植物 1,404 种, 其中木本植物481种, 草本植物859种, 藤本植物64种。植物beta 多样性随岛间距离和离岸距离差的增大而显著增加 $(P<0.001)$; 海岛面积和气候要素对植物beta多样性无显著影 响 $(P>0.05)$ 。岛间距离单独对beta多样性总变异的解释度为 $29.3 \%$, 离岸距离独立解释了 $2.8 \%$, 面积和气候共同解 释了 $0.5 \%$ 。 木本植物与草本植物的beta多样性格局与总体一致, 距离因子对木本植物beta多样性的解释度高于草本 植物(37.5\% > 25.3\%)。综上, 海岛植物beta多样性主要受岛间距离和离岸距离的影响, 反映了扩散限制是塑造中国 东部海岛植物beta多样性格局的主要生态过程。
\end{abstract}

关键词: 海岛面积; Jaccard指数; 岛间距离; 离岸距离; 气候; 扩散限制

\section{Beta diversity of vascular plants and its drivers in sea-islands of eastern China}

\author{
Xiangyu Liu ${ }^{1,2}$, Ciliang Zhao ${ }^{2}$, Mingshan Xu ${ }^{1,2}$, Qiming Liang ${ }^{1,2}$, Xiaotong Zhu ${ }^{1,2}$, Liang $\mathrm{Li}^{1,2}$, Enrong Yan ${ }^{1,2^{*}}$ \\ 1 School of Ecological and Environmental Sciences, East China Normal University, Shanghai 200241 \\ 2 Putuo Forest Ecosystem Research and Observation Station, Zhoushan, Zhejiang 316100
}

\begin{abstract}
Plant beta diversity describes how species composition shifts along environmental gradients. Islands are characterized by natural boundaries, variation in size and distance from the mainland, and they also experience intensive shifts in environmental properties. To date, plant beta diversity and its association with influencing factors across islands remain poorly understood. This study examined patterns and abiotic drivers of vascular plant beta diversity across 36 sea-islands in eastern China. Beta diversity was quantified using the Jaccard dissimilarity index based on presence/absence data, and patterns and drivers were analyzed by using partial Mantel tests and the variance partitioning approach. In total, there were 1,404 vascular plant species, including 481 woody species, 859 herbaceous species and 64 liana species. Plant beta diversity increased significantly with increase in cross-island distance and distance difference from the mainland. Differences in both island area and climatic factors did not affect plant beta diversity significantly. Cross-island distance accounted for $29.3 \%$ of total variation in plant beta diversity. Distance from the mainland explained 2.8\%, and island area and climatic differences together explained $0.5 \%$. The pattern of plant beta diversity was consistent between woody and herbaceous plants, but distance-based variables accounted for more beta diversity variation for woody than herbaceous plants (37.5\% > 25.3\%). Collectively, cross-island distance and the difference between islands in distance from the mainland are the main drivers of plant beta diversity. This suggests that dispersal limitation plays a key role in shaping plant beta diversity in the sea-islands of eastern China.
\end{abstract}

收稿日期: 2018-09-06; 接受日期: 2019-04-24

基金项目: 舟山市科技计划项目(2016C31031)和国家自然科学基金(31770467)

* 通讯作者 Author for correspondence. E-mail: eryan@des.ecnu.edu.cn 
Key words: island area; Jaccard index; cross-island distances; distances from the mainland; climate; dispersal limitation

Beta多样性反映了不同时空尺度上物种组成的 差异(Whittaker, 1960), 了解其时空格局不仅能为生 态学、生物地理学以及进化生物学等领域的关键问 题提供解释途径(Moritz et al, 2001), 还可以指导自 然保护区的设计和区域物种多样性的保护(Socolar et al, 2016)。近20年来, 聚焦于不同的时间和空间尺 度, 有关beta多样性的研究迅速增加(Gomzález-Megías et al, 2008; Fattorini, 2010; Zellweger et al, 2017; Legendre, 2019)。

目前, 关于beta多样性形成机制的基本共识是 其受生态位过程和扩散过程的共同影响(陈圣宾等, 2010)。生态位过程是指物种适应于特定环境产生专 性生态位, 因此环境梯度对物种的分布有分选作用 (species sorting), 即两个群落或区域的beta多样性 是二者间环境差异的结果。通常, 环境越相似, beta 多样性越低(Whittaker, 1960; MacArthur, 1965; Qian \& Ricklefs, 2007; Xing \& He, 2018)。扩散过程是指 beta多样性取决于群落或区域间的隔离程度和生物 类群的扩散能力, 即当隔离程度越低或物种扩散能 力越强时, beta多样性越低(Green \& Ostling, 2003; Fattorini, 2010; Catano et al, 2017)。检验这两个过程 在驱动不同生物类群beta多样性形成中的相对重要 性, 依然是生物多样性领域的研究热点之一。

大量关于不同植物类群种子扩散特征的研究 表明, 不同生活型的植物往往具有不同的扩散方式 (Westoby et al, 1996; 俞篗押等, 2018)。例如, 干旱 区草本植物以风力传播为主; 在亚热带森林中, 主 要木本植物依靠动物传播(Guitián \& Sánchez, 1992; 李娟等, 2013; 蒋裕良等, 2017)。考虑到扩散过程对 大尺度多样性格局的影响尤为明显(Qian \& Ricklefs, 2007; Vellend, 2016), 针对不同生活型的植物, 可以 合理推测: 在其他条件一致的情形下, 相对于草本, 扩散过程对木本植物的制约强度更大。

以往关于植物beta多样性的研究大多集中于大 陆生境, 关于海岛植物beta多样性格局及其影响因 素的报道较少。海岛作为相对孤立的地理单元, 为 检验生物类群beta多样性的影响因素, 特别是扩散 过程的相对重要性, 提供了绝佳的自然场所。同时,
大陆种库是陆桥型海岛的重要物种组成来源, 离岸 距离显著影响着物种的迁入、迁出等过程 (MacArthur \& Wilson, 1967), 进而会导致海岛物种 组成上的差异。因此, 海岛植物beta多样性可能不仅 会表现出与大陆连续生境相似的格局, 也可能有别 于大陆。

本文以中国东部36个海岛为研究对象, 利用维 管植物物种名录数据, 主要探讨以下 2 个科学问题: (1)中国东部海岛维管植物的beta多样性特征如何? 扩散过程是否是其主要的生态过程? (2)不同生活型 植物的beta多样性格局有何差异? 是否木本植物的 beta多样性高于草本植物? 且扩散过程对木本植物 beta多样性的解释度更高? 本文拟通过回答上述问 题, 为揭示中国东部海岛维管植物beta多样性的维 持格局提供依据。

\section{材料与方法}

\section{1 研究地点}

研究对象包括中国东部海域 $\left(117^{\circ} 25^{\prime} 12^{\prime \prime}-\right.$

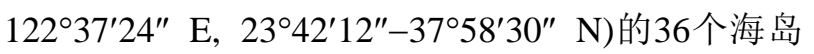
(图1), 南北跨越14个纬度, 其中 12 个海岛位于温带 季风气候区, 24 个海岛位于亚热带季风气候区。纬 度最高的为山东省的北长山岛, 纬度最低的为福建 省的东山岛。面积最大的为福建省的平潭岛 $\left(267.130 \mathrm{~km}^{2}\right)$, 面积最小的为江苏省的开山岛 $\left(0.016 \mathrm{~km}^{2}\right)$ 。离岸距离最远的为浙江省的外马廊山 岛 $(63.5 \mathrm{~km})$, 最近的则为江苏省的羊山岛 $(0.1 \mathrm{~km})$ (中国海岛志编纂委员会, 2013)。

\section{2 数据集}

海岛维管植物物种数据来源于已发表的海岛 资源综合调查研究报告、海岛植物区系调查文献, 以及2015-2018年间本实验室的实地调查(附录1)。 某种植物出现在海岛上, 记为“ “1”, 未出现则记为 “0”。采用 R 3.5.0软件中的plantlist程序包(Zhang, 2018)剔除栽培种。

为了检验环境过滤等生态位过程对海岛维管 植物beta多样性的影响, 我们收集了各海岛的年均 温、年均降水量以及年均风速数据, 并计算海岛间以 


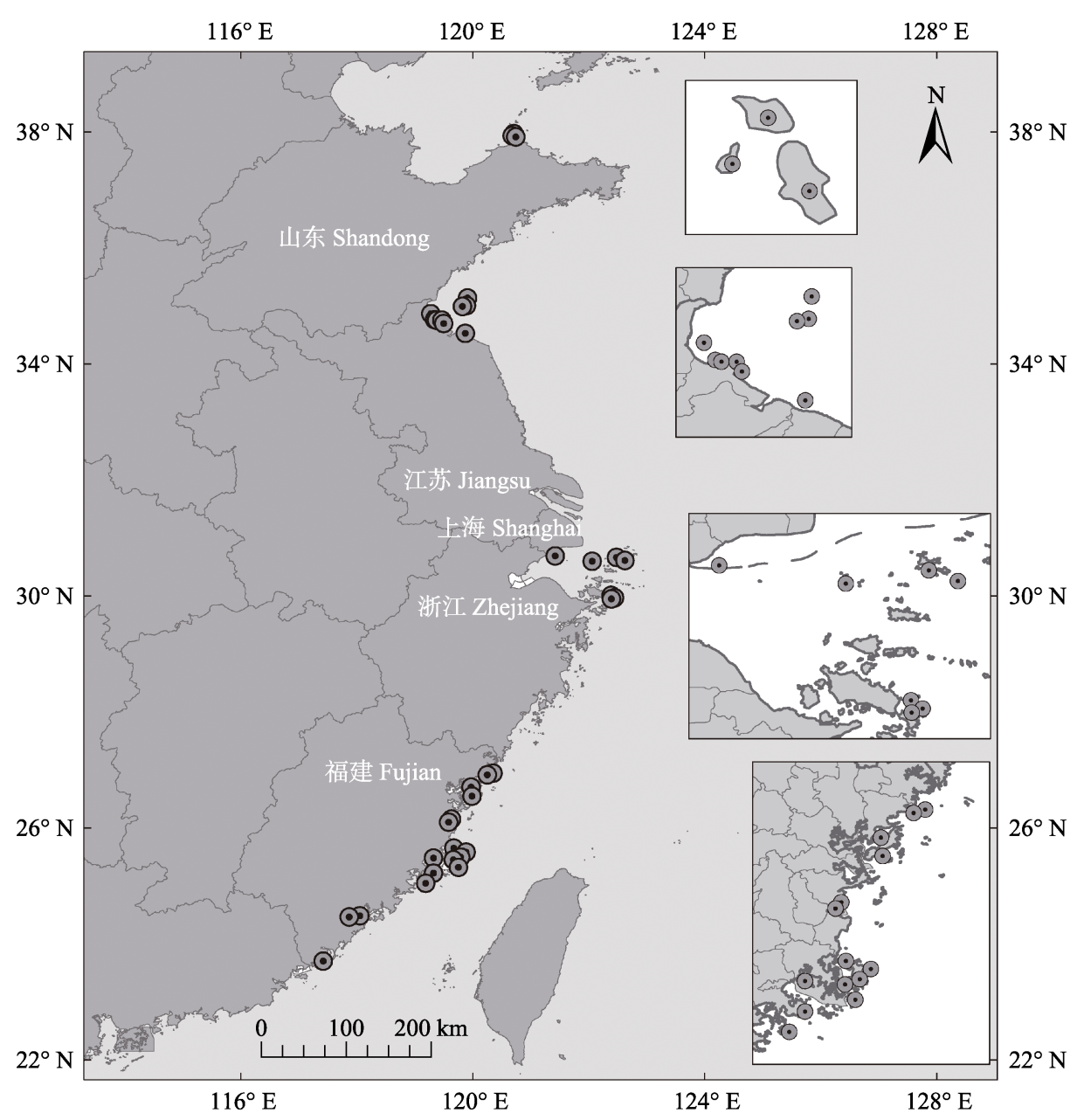

图1 中国东部36个研究海岛的分布图

Fig. 1 Locations of the 36 studied sea-islands in eastern China

上因子的马氏距离(Mahalanobis distance)来表征长 期影响海岛植物分布的气候因子的差异。以上数据 均摘自《中国海岛志》(中国海岛志编纂委员会, 2013) 及相关研究论文(附录1)。为了检验扩散过程对海岛 维管植物beta多样性的影响, 我们计算了各海岛的 离岸距离(海岛与最近大陆的距离)和海岛间的实际 空间距离(岛间距离)。海岛离岸距离通过Google Earth软件的ruler工具估计得到(Ibanez et al, 2018)。 岛间距离通过R 3.5.0中的geosphere程序包(Hijmans et al, 2011)基于经纬度计算得到。

\section{3 数据处理}

本文以Jaccard相异性指数来度量海岛维管植 物的beta多样性(Jaccard, 1912), 计算公式为:

$$
C_{\mathrm{j}}=1-a /(a+b+c)
$$

式中, $a$ 代表两个岛屿共有的物种数, $b$ 是仅存在于 第 1 个岛屿的物种数, $c$ 是仅存在于第 2 个岛屿的物
种数。Jaccard相异性指数越大, 表明物种组成的差 异越大。

采用Mantel偏相关方法检验海岛成对Jaccard相 异性指数(pairwise Jaccard dissimilarity)与岛间距 离、离岸距离差(两个海岛与最近大陆距离的差)、 面积差与气候差异的关系。在检验某个因子与成对 Jaccard相异性指数的关系时，需控制其他因子对两 者的协变影响，得到该因子与beta多样性被滤除协 变效应后的残差部分的关系。同时，由于海岛间的 成对 Jaccard相异性指数具有自相关性(Diserud \& Ødegaard, 2007), 本研究没有以成对Jaccard相异性 指数的平均值代表海岛整体beta多样性，而是以多 点Jaccard相异性指数 (multiple-site dissimilarity) 比 较不同生活型植物的beta多样性(Baselga et al, 2007; Baselga, 2013)。最后，为进一步探究生态位和扩散 过程对 beta多样性格局的影响, 采用变异分解 
(variance partitioning)的方法, 计算岛间距离、离岸 距离、面积和气候因子对beta多样性的相对贡献率 (Yang et al, 2015)。以上数据分析在R 3.5.0的ppcor (Kim, 2015)、 ecodist (Goslee \& Urban, 2007)、 betapart (Baselga \& Orme, 2012)以及vegan (Oksanen et al, 2012)程序包中完成。

\section{2 结果}

\section{1 海岛维管植物的物种丰富度}

36 个海岛共记录维管植物 1,404 种, 其中木本 植物481种, 草本植物859种, 藤本植物64种。木本 植物以豆科(42种)、蓄薇科(40种)为主, 草本植物以 禾本科(162种)、菊科(107种)和莎草科(52种)为主, 藤本植物多集中于葡萄科(15种)和毛莨科(9种)。物 种丰富度最高的海岛为福建省的粗芦岛, 共记录到
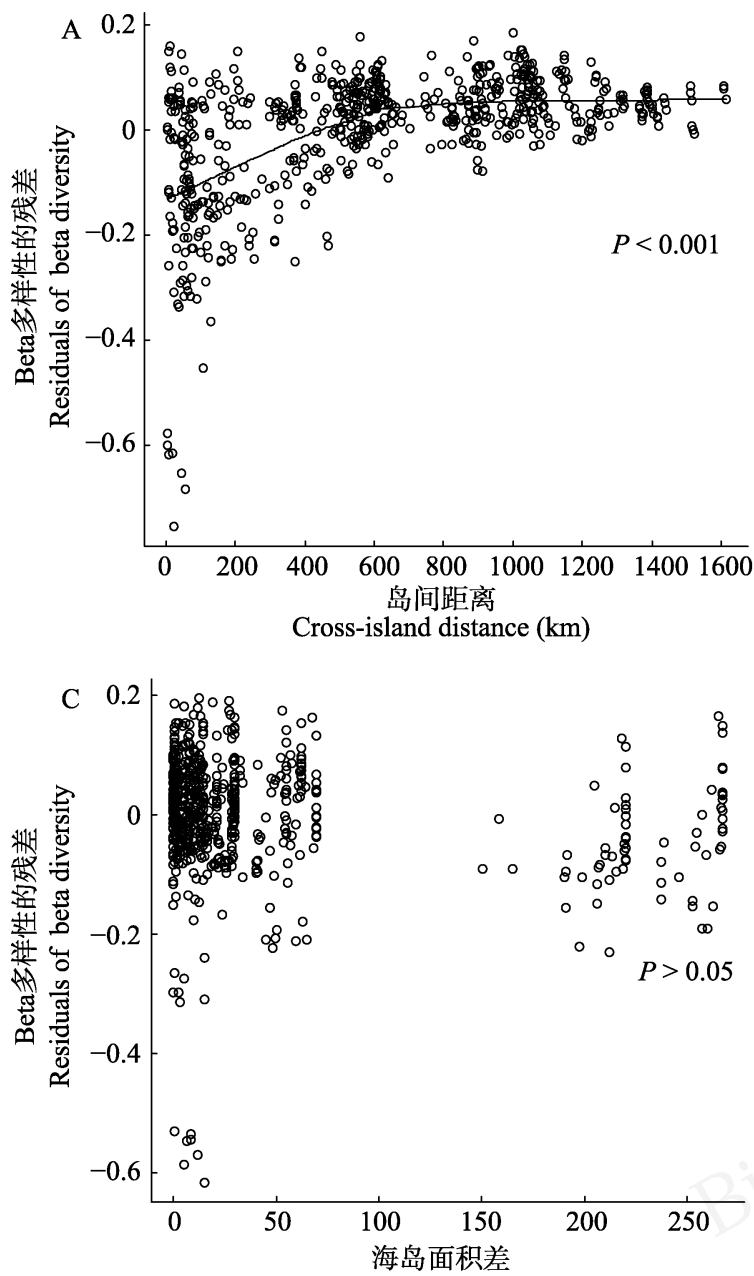

Area difference between islands $\left(\mathrm{km}^{2}\right)$
507种植物，最低的是江苏省的开山岛，仅记录到 30 种植物。

\section{2 海岛维管植物的beta多样性格局及其驱动因素}

Mantel偏相关分析表明，维管植物beta多样性 随岛间距离增加而显著增加 $(P<0.001)$, 随海岛离 岸距离差的增大显著上升 $(P<0.001)$ ，而与岛间面 积差 $(P>0.05)$ 和气候差异无关 $(P>0.05)$ (图2)。Beta 多样性的变异分解显示, 岛间距离和离岸距离分别 独立解释了植物 beta多样性总体变异的 $29.3 \%$ 和 $2.8 \%$, 海岛面积和气候因子共同解释了总变异的 $0.5 \%$ ，全部交互项解释了 $10.7 \%$ ，未解释的部分达 $56.7 \%$ (图3)。

\section{3 不同生活型植物beta多样性格局的比较}

木本与草本植物的beta多样性均随岛间距离和 离岸距离差的增加而显著增加 $(P<0.001)$, 而与海
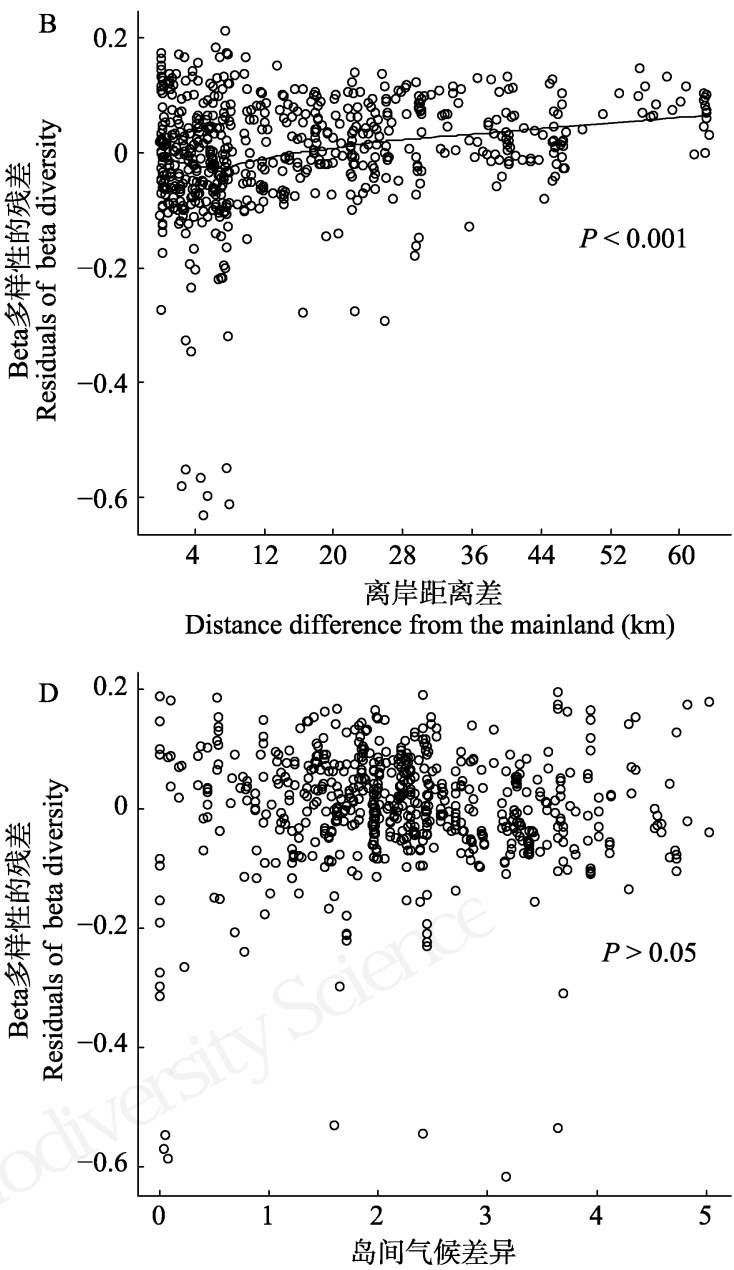

Climatic difference between islands

图2 维管植物beta多样性与岛间距离(A)、离岸距离差(B)、海岛面积差(C)以及岛间气候差异(D)的Mantel偏相关性

Fig. 2 The partial Mantel correlations of vascular plant beta diversity with cross-island distance, distance difference from the mainland, area difference and climatic difference between islands. 
表1 木本植物与草本植物的beta多样性与岛间距离、离岸距离差、面积差和气候差异的Mantel偏相关系数

Table 1 The partial Mantel correlation coefficients of beta diversity in woody and herbaceous plants with each of cross-island distance, distance difference from the mainland, area difference and climatic difference between islands

\begin{tabular}{lllll}
\hline $\begin{array}{l}\text { 植物生活型 } \\
\text { Plant life form }\end{array}$ & $\begin{array}{l}\text { 岛间距离 } \\
\text { Cross-island distance }\end{array}$ & $\begin{array}{l}\text { 离岸距离差 } \\
\text { Distance difference } \\
\text { from the mainland }\end{array}$ & $\begin{array}{l}\text { 面积差 } \\
\text { Area difference } \\
\text { between islands }\end{array}$ & $\begin{array}{l}\text { 气候差异 } \\
\text { Climatic difference } \\
\text { between islands }\end{array}$ \\
\hline $\begin{array}{l}\text { 草本植物 Herbaceous plants } \\
\text { 木本植物 Woody plants }\end{array}$ & $0.50^{* * * *}$ & $0.21^{* * * *}$ & -0.10 & 0.03 \\
\hline
\end{tabular}

*** $P<0.001$

岛面积差与气候差异无关 $(P>0.05)$ (表1)。根据36 个海岛木本和草本植物的多点Jaccard相异性指数, 两者的beta多样性均为 0.969 , 表明生活型对植物 beta多样性本身无显著影响。

Beta多样性变异分解表明, 岛间距离和离岸距 离分别解释了木本植物beta多样性总变异的 $33.7 \%$ 和 3.8\%, 草本植物 beta多样性总变异的 $23.1 \%$ 和 $2.2 \%$ (图3)。面积和气候共同解释了木本植物beta 多样性总变异的 $0.8 \%$, 草本植物总变异的 $0.3 \%$ 。全 部交互项解释了两者总变异的 $8.6 \%$ 和 $9.3 \%$ 。以上结 果表明距离因子对木本植物beta多样性的解释度高 于草本植物(37.5\% > 25.3\%)。

\section{3 讨论}

3.1 中国东部海岛维管植物beta多样性的生物地 理格局

本研究通过分析中国东部36个海岛维管植物

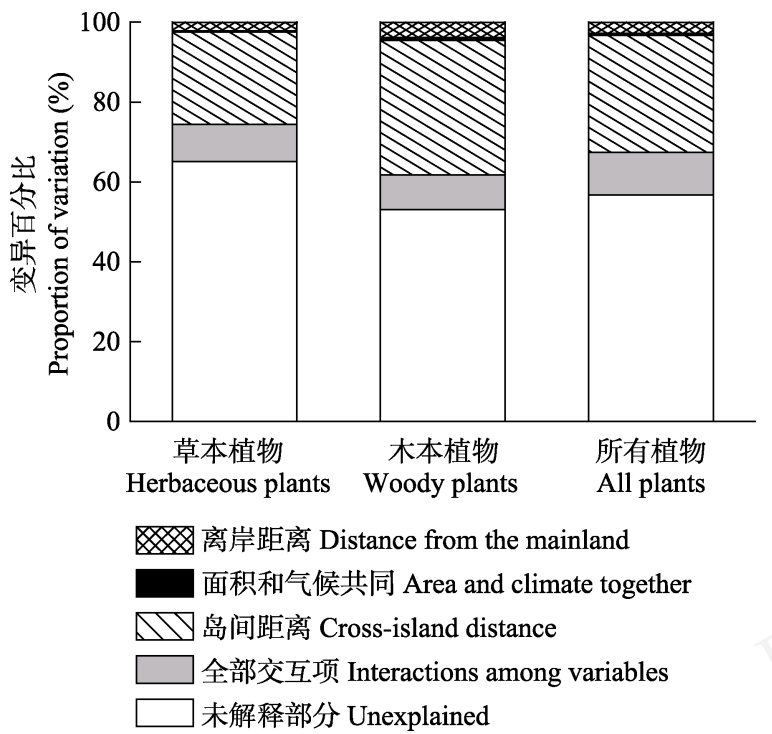

图3 中国东部海岛植物beta多样性的变异分解

Fig. 3 Variance decomposition of plant beta diversity across sea-islands in eastern China beta多样性的格局发现，岛间距离和海岛离岸距离 差均与植物beta多样性正相关。当海岛间的空间距 离增大时，岛屿间的物种交换率下降，不同海岛的 物种组成差异会更高, 因此beta多样性随岛间距离 增加而上升，此结果与其他研究基本一致(Qian, 2009; Bahram et al, 2013; Kubota et al, 2014; Xing \& He, 2018)。离岸距离代表了海岛的隔离程度, 对近 岸岛而言，大陆的物种迁入率更高，对远岸岛来说 则相反。因此, 当两个海岛的离岸距离差越小时, 其物种迁入率和迁出率基本相似，那么其物种组成 差异较小。相反, 两个海岛的离岸距离差越大时, 意味着两者间的物种迁入和迁出率差异越大，从而 导致岛屿局域物种库组成的不同，因此beta多样性 随离岸距离差增加而上升。这种现象也在其他岛屿 研究中得到印证, 例如, 在千岛湖陆桥岛屿中, 岛 屿到大陆的最小距离对植物beta多样性的形成具有 重要作用(彭思羿等, 2014)。在日本群岛，岛屿空间 距离和海峡障碍(隔离)是驱动植物beta多样性变化 格局的主要因素(Kubota et al, 2014)。

本研究也发现, 海岛维管植物的beta多样性与 海岛面积差无关，这一结果没有支持以往的研究观 点。主要原因可能有两点: (1)本文所研究海岛的面 积变异程度不均一, 绝大多数海岛面积集中在 $1 \mathrm{~km}^{2}$, 个别面积超过 $50 \mathrm{~km}^{2}$ ，中等面积的岛屿非常稀少， 这样，海岛间的面积差没有表现出明显的梯度变化 特征(图2C), 从而削弱了植物beta多样性与岛屿面 积差的关系。(2)我国东部海岛绝大多数为近岸岛, 人类干扰频繁, 一定程度上可能改变了岛屿植物的 beta多样性格局。越来越多的研究表明，人类活动可 显著改变传统岛屿生物地理学理论所预测的生物 多样性格局(Whittaker, 1995; Jesse et al, 2018)。人类 活动主要通过土地利用改变而弱化了岛屿的面积 属性, 从而模糊了岛屿物种数-面积关系(Kardol et 
al, 2018)。面积是经典岛屿生物地理学理论成立的 核心条件。然而, 人类的土地利用导致大型岛屿生 境的高度片断化或破碎化，一方面造成土著物种小 种群的局部灭绝和物种数的下降(Newbold et al, 2015), 另一方面通过大量新物种的引入, 使得海岛 物种库重新“洗牌”(van Kleunen et al, 2015)。例如, 人类干预使得太平洋岛屿上的植物种类在过去 100 年较之前增加了2倍之多(Sax \& Gaines, 2008)。

\section{2 海岛木本和草本植物beta多样性格局的差异}

对两种生活型植物beta多样性的变异分解的结 果表明, 岛间距离和离岸距离对木本植物beta多样 性的解释度均高于草本植物, 间接反映了木本植物 种类组成在岛间的差异更易受扩散限制的影响。在 本研究所记录物种的扩散方式中(未发表数据), 草 本植物中风力与鸟类传播方式所占比例高达 $46.3 \%$, 卡方检验显示, 其显著高于上述传播方式在木本植 物中的比例(33.9\%)。以往的研究发现, 物种beta多 样性与扩散能力存在一定的负相关关系(Bell, 2001; Green \& Ostling, 2003), 种间扩散能力差异越大, beta多样性差异越大(Gómez-Rodríguez et al, 2019)。 在本研究中, 虽然木本与草本植物的beta多样性在 海岛间无显著差异, 但上述观点可从岛间距离和离 岸距离对木本植物beta多样性更高的解释率上得到 间接证明。

值得指出的是, 本研究发现海岛面积和气候对 木本和草本植物单独和总体维管植物beta多样性的 解释度均较低。Kubota等(2014)也发现, 年均降水量 等环境因子与日本群岛木本植物的beta多样性无 关。尽管如此, 也不能绝对排除生态位过程(环境过 滤)在中国东部海岛植物beta多样性格局形成中的 作用。可能的原因是: 本研究所分析气候因子的年 际均值忽略了海岛内潜在的环境异质性和极端气 候。土壤基质是决定植物分布的重要因素(Itoh et al, 2003; Xing \& He, 2018), 补充土壤信息及其他代表 岛内环境异质性的因子, 有助于提出更具说服力的 结论。综上所述, 本研究为揭示中国东部海岛维管 植物的生物地理格局提供了部分证据, 有助于深入 理解海岛植物多样性的维持特征和潜在生态机制, 也为中国东部海岛的植物多样性保护工作提供了 一定理论基础。
致谢: 华东师范大学苏田、郑丽婷、郭超、妥涁以 及Umar Aftab等同学在野外植物调查中鼎力相助, 何东博士和高志文在数据分析和论文撰写中提供 了宝贵建议，黔南民族师范学院俞签押老师帮助校 对植物扩散方式，同时三位审稿专家对本文的初稿 提出建设性修改意见，在此表示感谢!

\section{参考文献}

Bahram M, Kõljalg U, Courty PE, Diédhiou AG, Kjøller R, Põlme S, Ryberg M, Veldre V, Tedersoo L (2013) The distance decay of similarity in communities of ectomycorrhizal fungi in different ecosystems and scales. Journal of Ecology, 1335-1344.

Baselga A (2013) Multiple site dissimilarity quantifies compositional heterogeneity among several sites, while average pairwise dissimilarity may be misleading. Ecography, 36, 124-128.

Baselga A, Jiménez-Valverde A, Niccolini G (2007) A multiple-site similarity measure independent of richness. Biology Letters, 3, 642-645.

Baselga A, Orme CDL (2012) betapart: An R package for the study of beta diversity. Methods in Ecology and Evolution, 3, 808-812.

Bell G (2001) Neutral marcoecology. Science, 293, 2413-2418.

Catano CP, Dickson TL, Myers JA (2017) Dispersal and neutral sampling mediate contingent effects of disturbance on plant beta-diversity: A meta-analysis. Ecology Letters, 20, 347-356.

Chen SB, Ouyang ZY, Xu WH, Xiao Y (2010) A review of beta diversity studies. Biodiversity Science, 18, 323-335. (in Chinese with English abstract) [陈圣宾, 欧阳志云, 徐卫 华, 肖炏 (2010) Beta多样性研究进展. 生物多样性, 18, 323-335.]

Diserud OH, Ødegaard F (2007) A multiple-site similarity measure. Biology Letters, 3, 20-22.

Fattorini S (2010) The influence of geographical and ecological factors on island beta diversity patterns. Journal of Biogeography, 37, 1061-1070.

Gómez-Rodríguez C, Miller KE, Castillejo J, Iglesias-Piñeiro J, Baselga A (2019) Understanding dispersal limitation through the assessment of diversity patterns across phylogenetic scales below the species level. Global Ecology and Biogeography, 28, 353-364.

Gomzález-Megías A, Menéndez R, Roy D, Brereton $\mathrm{T}$, Thomas CD (2008) Changes in the composition of British butterfly assemblages over two decades. Global Change Biology, 14, 1464-1474.

Goslee SC, Urban DL (2007) The ecodist package for dissimilarity-based analysis of ecological data. Journal of Statistical Software, 22(7), 1-19.

Green JL, Ostling A (2003) Endemics-area relationships: The 
influence of species dominance and spatial aggregation. Ecology, 84, 3090-3097.

Guitián J, Sánchez JM (1992) Seed dispersal spectra of plant communities in the Iberian Peninsula. Vegetatio, 98, 157-164.

Hijmans RJ, Williams E, Vennes C (2011) Geosphere: Spherical Trigonometry for Geographic Applications. http://cran.r-project. org/web/packages = geosphere. (accessed on 2017-11-05)

Ibanez T, Keppel G, Baider C, Birkinshaw C, Culmsee H, Cordell S, Florens FBV, Franklin J, Giardina CP, Gillespie TW, Laidlaw M, Litton CM, Martin TG, Ostertag R, Parthasarathy N, Randrianaivo R, Randrianjanahary M, Rajkumar M, Rasingam L, Ratovoson F, Reza L, Sack L, Aiba S, Webb E, Whitfeld TJS, Zang R, Birnbaum P (2018) Regional forcing explains local species diversity and turnover on tropical islands. Global Ecology and Biogeography, 27, 474-486.

Itoh A, Yamakura T, Ohkubo T, Kanzaki M, Palmiotto PA, LaFrankie JV, Ashton PS, Lee HS (2003) Importance of topography and soil texture in the spatial distribution of two sympatric dipterocarp trees in a Bornean rainforest. Ecological Research, 18, 307-320.

Jaccard P (1912) The distribution of the flora in the alpine zone. New Phytologist, 11, 37-50.

Jesse WAM, Behm JE, Helmus MR, Ellers J (2018) Human land use promotes the abundance and diversity of exotic species on Caribbean islands. Global Change Biology, 24, 4784-4796.

Jiang YL, Li XK, Guo YL, Ding T, Wang B, Xiang WS (2017) Diversity of climbing seed plants and their reproductive habit in a karst seasonal rain forest in Nonggang, Guangxi, China. Chinese Journal of Plant Ecology, 41, 716-728. (in Chinese with English abstract) [蒋裕良, 李先琨, 郭屹立, 丁涛, 王斌, 向悟生 (2017) 广西弄岗喀斯特季节性雨林 藤本种子植物多样性及繁殖习性. 植物生态学报, 41, 716-728.]

Kardol P, Fanin N, Wardle DA (2018) Long-term effects of species loss on community properties across contrasting ecosystems. Nature, 557, 710-713.

Kim S (2015) ppcor: An R package for a fast calculation to semi-partial correlation coefficients. Communications for Statistical Applications and Methods, 22, 665-674.

Kubota Y, Hirao T, Fujii S, Shiono T, Kusumoto B (2014) Beta diversity of woody plants in the Japanese archipelago: The roles of geohistorical and ecological processes. Journal of Biogeography, 41, 1267-1276.

Legendre P (2019) A temporal beta-diversity index to identify sites that have changed in exceptional ways in space-time surveys. Ecology and Evolution, 9, 3500-3514.

Li J, Guo C, Xiao ZS (2013) Fruit composition and seed dispersal strategies of woody plants in a Dujiangyan subtropical forest, Southwest China. Biodiversity Science, 21, 572-581. (in Chinese with English abstract) [李娟, 郭
聪，肖治术 (2013) 都江堰亚热带森林常见木本植物果 实组成与种子扩散策略. 生物多样性, 21, 572-581.]

MacArthur RH (1965) Patterns of species diversity. Biological Reviews, 40, 510-533.

MacArthur RH, Wilson EO (1967) The Theory of Island Biogeography. Princeton University Press, Princeton.

Moritz C, Richardson KS, Ferrier S, Monteith GB, Stanisic J, Williams SE, Whiffin T (2001) Biogeographical concordance and efficiency of taxon indicators for establishing conservation priority in a tropical rainforest biota. Proceedings of the Royal Society B: Biological Sciences, 268, 1875-1881.

Newbold T, Hudson LN, Hill SLL, Contu S, Lysenko I, Senior RA, Börger L, Bennett DJ, Choimes A, Collen B, Day J, De Palma A, Díaz S, Echeverria-Londoño S, Edgar MJ, Feldman A, Garon M, Harrison MLK, Alhusseini T, Ingram DJ, Itescu Y, Kattge J, Kemp V, Kirkpatrick L, Kleyer M, Correia DLP, Martin CD, Meiri S, Novosolov M, Pan Y, Phillips HRP, Purves DW, Robinson A, Simpson J, Tuck SL, Weiher E, White HJ, Ewers RM, Mace GM, Scharlemann JPW, Purvis A (2015) Global effects of land use on local terrestrial biodiversity. Nature, 520, 45-50.

Oksanen J, Blanchet FG, Kindt R, Wagnar HH (2012) vegan: Community Ecology Package. https://CRAN.R-project.org/ package=vegan. (accessed on 2019-02-04)

Peng SY, Hu G, Yu MJ (2014) Beta diversity of vascular plants and its influencing factors on islands in the Thousand Island Lake. Acta Ecologica Sinica, 34, 3866-3872. (in Chinese with English abstract) [彭思羿, 胡广, 于明坚 (2014) 千 岛湖岛屿维管植物 $\beta$ 多样性及其影响因素. 生态学报, 34, 3866-3872.]

Qian H (2009) Beta diversity in relation to dispersal ability for vascular plants in North America. Global Ecology and Biogeography, 18, 327-332.

Qian H, Ricklefs RE (2007) A latitudinal gradient in largescale beta diversity for vascular plants in North America. Ecology Letters, 10, 737-744.

Sax DF, Gaines SD (2008) Species invasions and extinction: The future of native biodiversity on islands. Proceedings of the National Academy of Sciences, USA, 105, 1149011497.

Socolar JB, Gilroy JJ, Kunin WE, Edwards DP (2016) How should beta-diversity inform biodiversity conservation? Trends in Ecology and Evolution, 31, 67-80.

The Editoral Committee of Sea Islands of China (2013) Sea Islands of China. China Ocean Press, Beijing. (in Chinese) [中国海岛志编纂委员会 (2013) 中国海岛志. 海洋出版 社, 北京.]

van Kleunen M, Dawson W, Essl F, Pergl J, Winter M, Weber E, Kreft H, Weigelt P, Kartesz J, Nishino M, Antonova LA, Barcelona JF, Cabezas FJ, Cárdenas D, Cárdenas-Toro J, Castaño N, Chacón E, Chatelain C, Ebel AL, Figueiredo E, Fuentes N, Groom QJ, Henderson L, Singh I, Kupriyanov A, 
Masciadri S, Meerman J, Morozova O, Moser D, Nickrent DL, Patzelt A, Pelser PB, Baptiste MP, Poopath M, Schulze M, Seebens H, Shu WS, Thomas J, Velayos M, Wieringa JJ, Pyšek P (2015) Global exchange and accumulation of non-native plants. Nature, 525, 100-103.

Vellend M (2016) Simulating dynamics in ecological communities. In: The Theory of Ecological Communities (eds Levin SA, Horn HS), pp. 76-96. Princeton University Press, Princeton.

Westoby M, Leishman M, Lord J, Poorter H, Schoen DJ (1996) Comparative ecology of seed size and dispersal. Philosophical Transactions of the Royal Society B: Biological Sciences, 351, 1309-1318.

Whittaker RH (1960) Vegetation of the Siskiyou Mountains, Oregon and California. Ecological Monographs, 30, 279-338.

Whittaker RJ (1995) Disturbed island ecology. Trends in Ecology and Evolution, 10, 421-425.

Xing DL, He FL (2018) Environmental filtering explains a U-shape latitudinal pattern in regional $\beta$-deviation for eastern North American trees. Ecology Letters, 22, 284-291.
Yang J, Swenson NG, Zhang GC, Ci XQ, Cao M, Sha LQ, Li J, Slik JWF, Lin LX (2015) Local-scale partitioning of functional and phylogenetic beta diversity in a tropical tree assemblage. Scientific Reports, 5, 12731.

Yu XY, Li YH, Yang GR (2018) Fruit types and seed dispersal modes of plants in different communities in Shilin Geopark, Yunnan, China. Chinese Journal of Plant Ecology, 42, 663-671. (in Chinese with English abstract) [俞䈗押, 李玉 辉, 杨光荣 (2018) 石林地质公园不同群落类型植物果 实组成与种子散布特征. 植物生态学报, 42, 663-671.]

Zellweger F, Roth T, Bugmann H, Bollmann K (2017) Beta diversity of plants, birds and butterflies is closely associated with climate and habitat structure. Global Ecology and Biogeography, 26, 898-906.

Zhang JL (2018) plantlist: Looking up the Status of Plant Scientific Names based on The Plant List Database. R package version 0.5.3. https://github.com/helixcn/plantlist/. (accessed on 2018-11-15)

(责任编委: 唐志尧 责任编辑: 间文杰)

\section{附录 Supplementary Material}

附录1 中国东部36个海岛的物种多样性及地理信息

Appendix 1 Species diversity and geographic information of the 36 sea-islands in eastern China http://www.biodiversity-science.net/fileup/PDF/2018235-1.pdf 
刘翔宇, 赵慈良, 许洺山, 梁启明, 朱晓粀, 李亮, 阎恩荣. 中国东部海岛维管植物的beta多样性及其驱动因 素. 生物多样性, 2019, 27 (4): 380-387.

http://www.biodiversity-science.net/CN/10.17520/biods.2018235

附录1 中国东部36个海岛的物种多样性及地理信息

Appendix 1 Species diversity and geographic information of the 36 sea-islands in eastern China

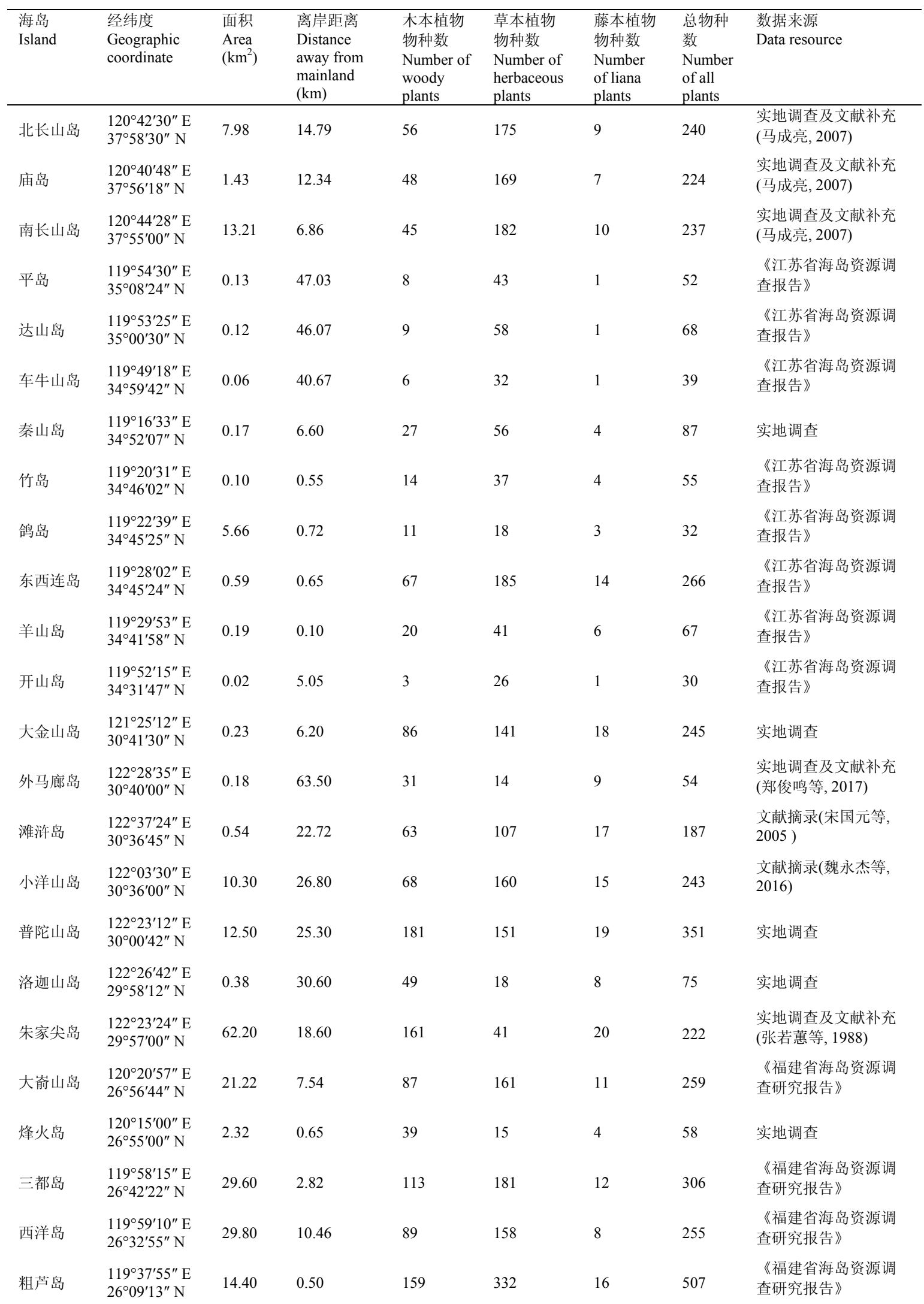


刘翔宇, 赵慈良, 许洺山, 梁启明, 朱晓粀, 李亮, 阎恩荣. 中国东部海岛维管植物的beta多样性及其驱动因 素. 生物多样性, 2019, 27 (4): 380-387.

http://www.biodiversity-science.net/CN/10.17520/biods.2018235

\begin{tabular}{|c|c|c|c|c|c|c|c|c|}
\hline 琅岐岛 & $\begin{array}{l}119^{\circ} 34^{\prime} 58^{\prime \prime} \mathrm{E} \\
26^{\circ} 06^{\prime} 01^{\prime \prime} \mathrm{N}\end{array}$ & 55.00 & 0.69 & 127 & 167 & 7 & 301 & $\begin{array}{l}\text { 《福建省海岛资源调 } \\
\text { 查研究报告》 }\end{array}$ \\
\hline 大练岛 & $\begin{array}{l}119^{\circ} 40^{\prime} 20^{\prime \prime} \mathrm{E} \\
25^{\circ} 39^{\prime} 10^{\prime \prime} \mathrm{N}\end{array}$ & 9.96 & 7.85 & 81 & 130 & 7 & 218 & $\begin{array}{l}\text { 《福建省海岛资源调 } \\
\text { 查研究报告》 }\end{array}$ \\
\hline 东痒岛 & $\begin{array}{l}119^{\circ} 53^{\prime} 07^{\prime \prime} \mathrm{E} \\
25^{\circ} 35^{\prime} 02^{\prime \prime} \mathrm{N}\end{array}$ & 4.80 & 30.27 & 57 & 121 & 4 & 182 & $\begin{array}{l}\text { 《福建省海岛资源调 } \\
\text { 查研究报告》 }\end{array}$ \\
\hline 海坛岛 & $\begin{array}{l}119^{\circ} 47^{\prime} 25^{\prime \prime} \mathrm{E} \\
25^{\circ} 29^{\prime} 48^{\prime \prime} \mathrm{N}\end{array}$ & 267.13 & 8.13 & 133 & 243 & 12 & 388 & $\begin{array}{l}\text { 实地调查及《福建省海 } \\
\text { 岛资源调查研究报告》 }\end{array}$ \\
\hline 江阴岛 & $\begin{array}{l}119^{\circ} 19^{\prime} 19^{\prime \prime} \mathrm{E} \\
25^{\circ} 28^{\prime} 53^{\prime \prime} \mathrm{N}\end{array}$ & 69.75 & 0.81 & 111 & 182 & 8 & 301 & $\begin{array}{l}\text { 《福建省海岛资源调 } \\
\text { 查研究报告》 }\end{array}$ \\
\hline 大屿岛 & $\begin{array}{l}119^{\circ} 39^{\prime} 51^{\prime \prime} \mathrm{E} \\
25^{\circ} 27^{\prime} 05^{\prime \prime} \mathrm{N}\end{array}$ & 0.22 & 1.80 & 36 & 62 & 7 & 105 & $\begin{array}{l}\text { 实地调查及文献摘录 } \\
\text { (陈慧等, 2016) }\end{array}$ \\
\hline 草屿岛 & $\begin{array}{l}119^{\circ} 45^{\prime} 09^{\prime \prime} \mathrm{E} \\
25^{\circ} 19^{\prime} 16^{\prime \prime} \mathrm{N}\end{array}$ & 7.05 & 4.30 & 75 & 141 & 6 & 222 & $\begin{array}{l}\text { 《福建省海岛资源调 } \\
\text { 查研究报告》 }\end{array}$ \\
\hline 南日岛 & $\begin{array}{l}119^{\circ} 19^{\prime} 19^{\prime \prime} \mathrm{E} \\
25^{\circ} 13^{\prime} 13^{\prime \prime} \mathrm{N}\end{array}$ & 29.60 & 8.33 & 104 & 340 & 15 & 459 & $\begin{array}{l}\text { 《福建省海岛资源调 } \\
\text { 查研究报告》 }\end{array}$ \\
\hline 湄洲岛 & $\begin{array}{l}119^{\circ} 11^{\prime} 16^{\prime \prime} \mathrm{E} \\
25^{\circ} 02^{\prime} 46^{\prime \prime} \mathrm{N}\end{array}$ & 14.35 & 3.37 & 100 & 331 & 12 & 443 & $\begin{array}{l}\text { 《福建省海岛资源调 } \\
\text { 查研究报告》 }\end{array}$ \\
\hline 大兔屿 & $\begin{array}{l}118^{\circ} 03^{\prime} 11^{\prime \prime} \mathrm{E} \\
24^{\circ} 29^{\prime} 07^{\prime \prime} \mathrm{N}\end{array}$ & 0.09 & 0.37 & 57 & 60 & 5 & 122 & $\begin{array}{l}\text { 文献摘录(张琳婷等, } \\
\text { 2017) }\end{array}$ \\
\hline 紫泥岛 & $\begin{array}{l}117^{\circ} 52^{\prime} 06^{\prime \prime} \mathrm{E} \\
24^{\circ} 27^{\prime} 54^{\prime \prime} \mathrm{N}\end{array}$ & 28.56 & 0.46 & 72 & 138 & 4 & 214 & $\begin{array}{l}\text { 《福建省海岛资源调 } \\
\text { 查研究报告》 }\end{array}$ \\
\hline 东山岛 & $\begin{array}{l}117^{\circ} 25^{\prime} 12^{\prime \prime} \mathrm{E} \\
23^{\circ} 42^{\prime} 12^{\prime \prime} \mathrm{N}\end{array}$ & 220.18 & 0.50 & 162 & 221 & 10 & 393 & $\begin{array}{l}\text { 《福建省海岛资源调 } \\
\text { 查研究报告》 }\end{array}$ \\
\hline
\end{tabular}

Chen H, Zhang LT, Ye ZY (2016) Investigation and landscape application of plant resource in Dayu Islet. Journal of Chinese Urban Forestry, 14(6), 18-23. (in Chinese with English abstract) [陈慧, 张琳婷, 叶志勇 (2016) 平潭大屿植物资源调查与园林应用的探讨. 中国城市林业, 14(6), 18-23.]

Leading Group Office of Jiangsu Province Island Resources Comprehensive Investigation (1996) Comprehensive Survey Report on Island Resources of Jiangsu Province. Scientific and Technical Documentation Press, Beijing. (in Chinese) [江苏省海岛资源综合调查领导小组办公室 (1996) 江苏省海岛资源综合调查报告. 科学技术文献出版社, 北京.]

Ma CL (2007) Studies on Flora and Community Structure of Changdao in Shandong. PhD dissertation, Nanjing Forestry University, Nanjing. (in Chinese with English abstract) [马成亮 (2007) 山东长岛列岛植物区系及 群落结构研究. 博士学位论文, 南京林业大学, 南京.]

Song GY, Cao T, Yao JX (2005) Study on flora of the Tanxu Island, Hangzhou Gulf. Guihaia, 25, 1-7. (in Chinese with English abstract) [宋国元，曹同，姚建新 (2005) 杭州湾滩浒岛种子植物区系的研究. 广西植物, 25, 1-7.]

The Committee of Fujian Province Island Resources Comprehensive Investigation (1996) Comprehensive Survey and Research Report on Island Resources of Fujian Province. China Ocean Press, Beijing. (in Chinese) [福建 省海岛资源综合调查委员会 (1996) 福建省海岛资源综合调查研究报告. 海洋出版社, 北京.]

Wei YJ, Yang YF, He QY, Cai YH, Song GY (2016) Distribution characteristics of plants and environmental factors analysis in Xiaoyangshan Island, Zhejiang. Journal of Shanghai Normal University (Natural Sciences), 45, 81-93. (in Chinese with English abstract) [魏永杰, 杨耀芳, 何琴燕, 蔡燕红, 宋国元 (2016) 浙江嵊 泗小洋山岛植物分布特点和环境因子分析. 上海师范大学学报(自然科学版), 45, 81-93.]

Zhang LT, Wang WQ, Jiang DG (2017) Analysis of seed flora and its remediation strategies on Xiamen Datu Island. Ocean Development and Management, (3), 81-87. (in Chinese with English abstract) [张琳婷, 王文 
刘翔宇, 赵慈良, 许洺山, 梁启明, 朱晓形, 李亮, 阎恩荣. 中国东部海岛维管植物的beta多样性及其驱动因 素. 生物多样性, 2019, 27 (4): 380-387.

http://www.biodiversity-science.net/CN/10.17520/biods.2018235

卿，姜德刚 (2017) 厦门大兔屿种子植物区系分析及其植被修复对策研究. 海洋开发与管理，(3), 81-87.]

Zhang RH, Zhou SL, Xu YL, Chen DZ (1988) Preliminary investigation of the forestry species on Taohua Island and Zhujiajian Island. Journal of Zhejiang Forestry College, 5, 145-166. (in Chinese with English abstract) [张若㥶, 周世良, 徐耀良, 陈大钊 (1988) 桃花岛及朱家尖岛森林植物的初步调查. 浙江林学院学报, 5, 145-166.]

Zheng JM, Fang X, Zhu XP, Zhu DD, Deng CY, Huang LJ (2017) Vegetation characteristics and plant diversity of Waimalangshan Island, Zhoushan. Guihaia, 37, 271-279. (in Chinese with English abstract) [郑俊鸣, 方笑, 朱雪平, 朱丹丹, 邓传远，黄柳菁 (2017) 外马廊山岛植被特性与植物多样性. 广西植物, 37, 271-279.] 